\title{
Neoclassical momentum transport in a collisional stellarator and a rippled tokamak
}

\author{
Andrei N Simakov \\ Theoretical Division, Los Alamos National Laboratory, \\ Los Alamos, New Mexico 87545, U.S.A. \\ Per Helander \\ Max-Planck-Institut für Plasmaphysik, EURATOM-Association, \\ 17491 Greifswald, Germany
}

(March 4, 2009)

\begin{abstract}
Short mean-free path two-fluid equations are employed to evaluate the lowest order non-ambipolar radial current in plasma confined by a three-dimensional toroidal magnetic field. The result is used to obtain a necessary condition for intrinsic ambipolarity of plasma transport in such a field and to derive a criterion for the importance of the toroidal field ripple for collisional tokamak plasma rotation. The ripple effects on toroidal plasma rotation are found to be negligible if the characteristic perpendicular length scale is determined by the pedestal width of order the poloidal ion gyroradius (as may be the case in the $\mathrm{H}$-mode regime), but may conceivably become important for more shallow plasma gradients.
\end{abstract}




\section{INTRODUCTION}

Plasma flow in magnetic confinement devices can strongly influence plasma stability and transport and is therefore important to understand. In particular, sheared flow is known to enhance particle and heat confinement by suppressing and regulating turbulent transport [1]. Understanding plasma flow requires knowledge of plasma momentum transport, both its turbulent and collisional (predominantly neoclassical) channels. The reason is that although radial variation of turbulence-driven zonal flow is often much faster than that of the global (predominantly neoclassical) flow, the amplitude of the former is normally much smaller than that of the latter. As a result, contributions to flow shear from both can in principle be important.

Momentum transport theory in an axisymmetric tokamak is rather involved and still incomplete, even in the turbulence-free neoclassical limit. The reason is that neoclassical plasma transport in this case is "intrinsically ambipolar" $[2,3]$ and therefore cross-field ion viscosity plays a fundamental role in the radial transport of toroidal angular momentum [4]. However, accounting for this viscosity component, including the so-called perpendicular viscosity, requires difficult-to-evaluate corrections of order $\delta^{2}(\nu / \Omega) \ll 1$ to the lowest order ion distribution function, which is a Maxwellian in the absence of strong external driving sources [5]. Here, $\delta \ll 1$ is the small ion gyroradius expansion parameter, $\Omega$ is the ion gyrofrequency, and $\nu \ll \Omega$ is the ion collision frequency. This problem was only recently solved completely for short mean-free path plasma [6-9]. At the same time, a complete understanding of neoclassical momentum transport in the long mean-free path or banana regime for the plasma rotation velocity small compared with the ion thermal speed still remains elusive since existing treatments $[10,11]$ only consider large-aspect-ratio circular-cross-section tokamak limit and arbitrarily neglect poloidal variations of plasma density, ion temperature, 
and electrostatic potential, which play a fundamental role [7,9]. On the other hand, if the rotation velocity is assumed large, i.e., comparable to the ion thermal speed, the banana-regime momentum transport problem becomes less complicated and has been solved completely $[12,13]$.

It is somewhat easier to understand neoclassical plasma momentum transport in a strongly-rippled tokamak or a stellarator [14] since in those cases, due to the confining magnetic field geometry, particle transport is not "intrinsically ambipolar" and momentum transport is dominated by the so-called parallel ion viscosity, which is described by an order $\delta$ correction to lowest order ion distribution function and is much easier to evaluate. In fact, it has been shown that neoclassical plasma transport and rotation in a three-dimensional toroidal magnetic field are equivalent to those in an axisymmetric tokamak if [15] and only if [16] the confining magnetic field is either quasi-axisymmetric [17] or quasi-helically symmetric [18]. On the other hand, the most difficult to understand experimentally-relevant situations are for a tokamak with small toroidal field ripple and for a quasi-symmetric stellarator with small helical field ripple. Depending on the ripple size, effects of both parallel and cross-field ion viscosities can be important and must be accounted for.

Since hot magnetized plasmas have long mean-free paths, most of the analytical work on momentum transport concentrates on the banana-plateau collisionality regime. Various aspects of the neoclassical momentum transport problem in threedimensional toroidal magnetic fields in this collisionality regime have been studied in Refs. [19-27] and other publications. A good overview of the earlier work is given in the review paper [28].

In this paper, we consider short mean-free path plasma confined by an arbitrary three-dimensional toroidal magnetic field and investigate effects of parallel ion viscosity on rotation of such plasma. Then, we apply the results to a tokamak with a small 
toroidal magnetic field ripple to determine what ripple size can affect the axisymmetric plasma rotation result [6-9]. The main purpose of this work is to gain understanding on the transition from a two-dimensional to a three-dimensional toroidal magnetic configurations. This problem is not new and has been studied in Refs. [29-34], but none of these treatments is completely satisfactory. As discussed in the Appendix of this paper, Ref. [29] contains an error in evaluation of radial plasma current due to parallel ion viscosity, arriving at the result that, contrary to the authors' claim, does not vanish in the axisymmetric tokamak or quasi-symmetric stellarator limits. Reference [30] only considered a large-aspect-ratio rippled tokamak and neglected cross-field ion viscosity, and was therefore unable to describe the axisymmetric limit and consequently the case of small toroidal field ripple. Calculations based on the moment approach to neoclassical theory $[31,35]$ cannot exactly recover the correct expression for the parallel ion flow velocity, which was first calculated by Hazeltine [36] in the axisymmetric tokamak case and in this paper for an arbitrary toroidal geometry. In addition, Refs. [32, 33] neglected temperature gradients and cross-field ion viscosity. On the other hand, Ref. [32] considered additional potentially important effects which are not accounted for herein, such as time evolution, presence of neutral particles, and external bias currents. Finally, Ref. [34] studied large-aspect-ratio three-dimensional toroidal magnetic confinement configurations with a circular crosssection and also neglected cross-field ion viscosity. In this work, we allow arbitrary aspect ratios and poloidal cross-sections, account for effects of temperature gradients and cross-field viscosity, and do not employ the moment approach.

This paper is organized as follows. Section II describes our orderings and assumptions. Lowest-order expressions for ion particle and heat fluxes in an arbitrary three-dimensional toroidal magnetic field are obtained in Sec. III, and are then used in Sec. IV to evaluate the non-ambipolar radial plasma current due to parallel ion 
viscosity and obtain a necessary condition for intrinsically ambipolar neoclassical particle transport in such a magnetic field. Section V applies the general expression to evaluate this non-ambipolar radial current in the case of a large-aspect-ratio tokamak with a small toroidal magnetic field ripple. Finally, this current is employed in Sec. VI to evaluate how large the ripple should be to make plasma rotation deviate from the axisymmetric limit [6-9]. Our conclusions are then presented in Sec. VII.

\section{ORDERINGS AND ASSUMPTIONS}

We consider a plasma consisting of electrons and singly-charged ions and adopt the standard neoclassical collisional transport ordering for the Pfirsch-Schlüter regime (see e.g. Refs. $[3,35])$. The primary expansion parameters are based on smallness of the ion gyroradius, $\rho_{i}=v_{T i} / \Omega$, and the ion mean-free path, $\lambda_{i}=v_{T i} / \nu$, as compared with the characteristic perpendicular, $L_{\perp}$, and parallel, $L_{\|}$, length scales, respectively:

$$
\begin{gathered}
\delta \equiv \frac{\rho_{i}}{L_{\perp}} \ll 1, \\
\Delta \equiv \frac{\lambda_{i}}{L_{\|}} \ll 1 .
\end{gathered}
$$

Here, $v_{T i}=\sqrt{2 T / M}$ is the ion thermal speed, with $T$ the ion temperature and $M$ the ion mass; $\Omega=e B /(M c)$ is the ion gyrofrequency, with $e$ the magnitude of the electron charge, $c$ the speed of light, and $B$ the magnitude of the magnetic field $\boldsymbol{B}$; and $\nu=4 \pi^{1 / 2} n e^{4} \ln \Lambda /\left(3 M^{1 / 2} T^{3 / 2}\right)$ is the ion collision frequency [37], with $n$ the plasma density and $\ln \Lambda$ the Coulomb logarithm. The parallel length scale can be comparable with or larger than the perpendicular length scale, but not too large: $L_{\perp} \lesssim L_{\|}<L_{\perp} / \delta$.

We employ drift-ordered short mean-free path expressions [38] for the ion viscosity that were obtained by assuming that the ion flow velocity, $\boldsymbol{V}$, is of the order of the diamagnetic drift velocity, which in turn is of the order of the ion diamagnetic heat 
flux, $\boldsymbol{q}_{d}$, divided by the ion pressure, $p=n T$ :

$$
\frac{|\boldsymbol{V}|}{v_{T i}} \sim \frac{\left|\boldsymbol{q}_{d}\right|}{p v_{T i}} \sim \delta
$$

In addition, $\nu / \Omega \ll 1$ is assumed.

As usual in transport theory, we also assume that the plasma density, $n$, the electron and the ion temperatures, $T$ and $T_{e}$, respectively, and the electrostatic potential, $\phi$, are flux functions in lowest order, so that the dominant variation within a flux surface is due to a variation of the magnetic field. In particular, we assume that

$$
\frac{B \cdot \nabla \ln T}{B \cdot \nabla \ln B} \ll 1
$$

It can be shown a posteriori that the left-hand side of this inequality is of order $\delta / \Delta$, so that the assumption

$$
\frac{\delta}{\Delta} \ll 1
$$

must be made.

The time scale of interest is assumed to be that associated with the collisional ion radial heat transport, namely

$$
\frac{\partial}{\partial t} \sim \frac{\chi_{T}}{L_{\perp}^{2}} \sim \frac{\nu \delta^{2}}{\iota^{2}}
$$

where $\chi_{T} \sim \nu \delta^{2} / \iota^{2}$ is the ion thermal diffusivity, with $\iota$ the rotational transform. This time scale is assumed to be much shorter than the characteristic time scale for the variation of the vector potential, $\boldsymbol{A}$, which is determined by the resistive diffusion of the magnetic field, so that $\beta(M / m)^{1 / 2} \gg \iota^{2}$, with $\beta \equiv 8 \pi n\left(T+T_{e}\right) / B^{2}$ and $m$ the electron mass. As a result, the electric field, $\boldsymbol{E}=-\boldsymbol{\nabla} \phi-c^{-1} \partial \boldsymbol{A} / \partial t$, is electrostatic to the order we require,

$$
\frac{c^{-1}|\partial \boldsymbol{A} / \partial t|}{|\boldsymbol{\nabla} \phi|} \ll \frac{\delta}{\Delta \iota^{2}} \ll 1,
$$

where we estimate $|\boldsymbol{A}| \sim B L_{\|}, e \phi \sim T_{e} \sim T$. 
Finally, we assume that $\boldsymbol{B}$ possesses three-dimensional nested toroidal flux surfaces labeled by poloidal flux $\chi$.

\section{LOWEST ORDER ION HEAT AND PARTICLE FLOWS}

In the calculation that follows we require lowest order expressions for the ion heat and particle flows. This section evaluates such expressions. To avoid employing a specific coordinate system we introduce a solenoidal vector field

$$
\boldsymbol{h} \equiv \frac{\hat{\boldsymbol{b}} \times \boldsymbol{\nabla} \chi}{B}+u \boldsymbol{B}
$$

where $\hat{\boldsymbol{b}} \equiv \boldsymbol{B} / B$ and $u$ satisfies

$$
\nabla_{\|} u=\frac{2}{B^{2}} \hat{\boldsymbol{b}} \times \nabla \chi \cdot \nabla \ln B,
$$

so that $\nabla \cdot \boldsymbol{h}=0$. The vector $\boldsymbol{h}$ is proportional to the equilibrium current, $\boldsymbol{J}_{0}$, satisfying $\nabla \cdot \boldsymbol{J}_{0}=0$ and $\boldsymbol{J}_{0} \times \boldsymbol{B}=c \nabla p_{\Sigma}$, where $p_{\Sigma}(\chi)$ is the sum of the ion and electron pressures: $\boldsymbol{J}_{0}=c\left(\mathrm{~d} p_{\Sigma} / \mathrm{d} \chi\right) \boldsymbol{h}$. In particular, the quantity $u$ is proportional to the parallel current divided by $B$ and defined up to an arbitrary flux function (recall that the standard Pfirsch-Schlüter current in an axisymmetric tokamak is also evaluated up to a flux function times $B$ ). It is easy to show a posteriori that none of the results in the remainder of this paper depend on the value of this flux function.

The lowest order ion heat flow is given by the sum of the parallel and the diamagnetic contributions,

$$
\boldsymbol{q}=q_{\|} \hat{\boldsymbol{b}}+\frac{5 c p}{2 e B} \hat{\boldsymbol{b}} \times \nabla T .
$$

Substituting this expression into the ion energy conservation equation, we obtain in lowest order

$$
\boldsymbol{\nabla} \cdot \boldsymbol{q}=\boldsymbol{B} \cdot \boldsymbol{\nabla}\left(\frac{q_{\|}}{B}-\frac{5 c p}{2 e} \frac{\partial T}{\partial \chi} u\right)=0
$$


resulting in

$$
q_{\|}=L(\chi) B+\frac{5 c p}{2 e} \frac{\partial T}{\partial \chi} u B
$$

Since $q_{\|}=-125 p /(32 M \nu) \nabla_{\|} T$, where $\nu=1 / \tau_{i}=4 \pi^{1 / 2} n e^{4} \ln \Lambda /\left(3 M^{1 / 2} T^{3 / 2}\right)$ is the inverse ion collision time [37], we have $\left\langle B q_{\|}\right\rangle=0$ if $\langle\cdots\rangle$ denotes a flux-surface average defined as a volume average between two neighboring flux surfaces. Using this constraint in Eq. (11) to evaluate $L(\chi)$ we finally obtain

$$
q_{\|}=\frac{5 c p}{2 e} \frac{\partial T}{\partial \chi}\left(u B-\frac{\left\langle u B^{2}\right\rangle}{\left\langle B^{2}\right\rangle} B\right)
$$

and

$$
\nabla_{\|} T=\frac{16}{25} \frac{\nu}{\Omega} \frac{\partial T}{\partial \chi}\left(\frac{\left\langle u B^{2}\right\rangle}{\left\langle B^{2}\right\rangle} B^{2}-u B^{2}\right) .
$$

The flux-surface averaged radial ion heat flux is then obtained from Eqs. (9) and (13) and becomes

$$
\begin{array}{r}
\langle\boldsymbol{q} \cdot \boldsymbol{\nabla} \chi\rangle=-\left\langle\frac{5 c p}{2 e B} \hat{\boldsymbol{b}} \times \boldsymbol{\nabla} \chi \cdot \boldsymbol{\nabla} T\right\rangle \approx \frac{5 c p}{2 e}\left\langle u B \nabla_{\|} T\right\rangle= \\
\frac{8}{5} \frac{\nu p}{M}\left(\frac{B}{\Omega}\right)^{2} \frac{\partial T}{\partial \chi}\left(\frac{\left\langle u B^{2}\right\rangle^{2}}{\left\langle B^{2}\right\rangle}-\left\langle u^{2} B^{2}\right\rangle\right) .
\end{array}
$$

Note that the flux-surface averaged heat conductivity defined by this equation must be positive by the Schwartz inequality, $\langle f g\rangle^{2} \leq\left\langle f^{2}\right\rangle\left\langle g^{2}\right\rangle$, valid for arbitrary real functions $f$ and $g$.

The standard axisymmetric limit of results (13) and (14) is recovered by noticing that for an axisymmetric tokamak with $\boldsymbol{B}=I(\chi) \boldsymbol{\nabla} \zeta+\boldsymbol{\nabla} \zeta \times \boldsymbol{\nabla} \chi$, where $\zeta$ is the negative of the ordinary cylindrical angle measured about the toroidal axis, $\left(2 / B^{2}\right) \hat{\boldsymbol{b}} \times$ $\nabla \chi \cdot \nabla \ln B=-\nabla_{\|}\left(I / B^{2}\right)$. Then, Eq. (8) gives

$$
\left.u\right|_{a s}=-\frac{I(\chi)}{B^{2}}+F_{1}(\chi)
$$

with $F_{1}(\chi)$ a flux function. Using this result in Eqs. (13) and (14) gives the usual 
axisymmetric Pfirsch-Schlüter expressions [3, 36]

$$
\begin{array}{r}
\left.\nabla_{\|} T\right|_{a s}=\frac{16}{25} \frac{\nu I}{\Omega} \frac{\partial T}{\partial \chi}\left(1-\frac{B^{2}}{\left\langle B^{2}\right\rangle}\right), \\
\left.\langle\boldsymbol{q} \cdot \nabla \chi\rangle\right|_{\text {as }}=\frac{8}{5} \frac{\nu p I^{2}}{M} \frac{B^{2}}{\Omega^{2}} \frac{\partial T}{\partial \chi}\left(\frac{1}{\left\langle B^{2}\right\rangle}-\left\langle\frac{1}{B^{2}}\right\rangle\right) .
\end{array}
$$

The lowest order ion flow is given by the sum of the parallel, the $\boldsymbol{E} \times \boldsymbol{B}$, and the diamagnetic contributions,

$$
n \boldsymbol{V}=n V_{\|} \hat{\boldsymbol{b}}+\frac{c}{e B} \hat{\boldsymbol{b}} \times(e n \boldsymbol{\nabla} \phi+\nabla p) .
$$

Using this expression in the ion continuity equation, we obtain in lowest order

$$
\boldsymbol{\nabla} \cdot(n \boldsymbol{V})=\boldsymbol{B} \cdot \boldsymbol{\nabla}\left[\frac{n V_{\|}}{B}-c n\left(\frac{\partial \phi}{\partial \chi}+\frac{1}{e n} \frac{\partial p}{\partial \chi}\right) u\right]=0,
$$

so that

$$
V_{\|}=\frac{B}{n} K(\chi)+c\left(\frac{\partial \phi}{\partial \chi}+\frac{1}{e n} \frac{\partial p}{\partial \chi}\right) u B
$$

As in the corresponding calculation for tokamaks [36], the unknown flux function $K(\chi)$ can be determined from the flux surface average of electron plus ion parallel momentum equation, which gives in lowest order

$$
\left\langle\boldsymbol{B} \cdot\left(\boldsymbol{\nabla} \cdot \overleftrightarrow{\pi}_{\|}\right)\right\rangle=-\left\langle\pi_{\|} \nabla_{\|} B\right\rangle=0
$$

The ion parallel viscosity, $\overleftrightarrow{\pi}_{\|}=(\hat{\boldsymbol{b}} \hat{\boldsymbol{b}}-\overleftrightarrow{I} / 3) \pi_{\|}$, with $\overleftrightarrow{I}$ the unit dyad, was obtained in Ref. [38]. The pressure anisotropy $\pi_{\|}=p_{\|}-p_{\perp}$ is given to the order required by [39]

$$
\begin{array}{r}
\pi_{\|}=\frac{c \eta B}{2}\left(\nabla_{\|} u\right)\left(\frac{\partial \phi}{\partial \chi}+\frac{1}{e n} \frac{\partial p}{\partial \chi}+\frac{1.61}{e} \frac{\partial T}{\partial \chi}\right) \\
-\frac{2 \eta}{\sqrt{B}} \nabla_{\|}\left(\sqrt{B} V_{\|}\right)-\frac{1.42 \eta}{p \sqrt{B}} \nabla_{\|}\left(\sqrt{B} q_{\|}\right),
\end{array}
$$

where $\eta \equiv 0.96 p / \nu$ is the ion parallel viscosity coefficient [37], and we employed Eq. (8). Using this $\pi_{\|}$in the constraint (19) we obtain

$$
\begin{array}{r}
K(\chi)=-\frac{c}{e} \frac{\left\langle\left(\nabla_{\|} \ln B\right) \nabla_{\|}\left(u B^{2}\right)\right\rangle}{2\left\langle\left(\nabla_{\|} B\right)^{2}\right\rangle}\left(e n \frac{\partial \phi}{\partial \chi}+\frac{\partial p}{\partial \chi}\right) \\
+\frac{c}{e} n \frac{\partial T}{\partial \chi}\left(1.77 \frac{\left\langle u B^{2}\right\rangle}{\left\langle B^{2}\right\rangle}-0.91 \frac{\left\langle\left(\nabla_{\|} \ln B\right) \nabla_{\|}\left(u B^{2}\right)\right\rangle}{\left\langle\left(\nabla_{\|} B\right)^{2}\right\rangle}+0.05 \frac{\left\langle u\left(\nabla_{\|} B\right)^{2}\right\rangle}{\left\langle\left(\nabla_{\|} B\right)^{2}\right\rangle}\right) .
\end{array}
$$


Employing expression (21) for $K(\chi)$ in Eq. (18) and considering the axisymmetric limit (15) recovers the standard axisymmetric Pfirsch-Schlüter expression [36]

$$
V_{\|}=-\frac{c I}{B}\left(\frac{\partial \phi}{\partial \chi}+\frac{1}{e n} \frac{\partial p}{\partial \chi}\right)-\frac{c I B}{e\left\langle B^{2}\right\rangle} \frac{\partial T}{\partial \chi}\left(1.77+0.05 \frac{\left\langle B^{2}\right\rangle\left\langle\left(\nabla_{\|} \ln B\right)^{2}\right\rangle}{\left\langle\left(\nabla_{\|} B\right)^{2}\right\rangle}\right) .
$$

Calculations based on the moment approach to neoclassical theory [31, 35] cannot exactly recover the correct expression for the parallel ion flow velocity (they miss the very last term in Eq. (22), though the error tends to be small in practice), which was first calculated by Hazeltine [36] in the axisymmetric tokamak case and in this paper for an arbitrary toroidal geometry.

\section{RADIAL CURRENT}

We can now use the lowest order ion heat and particle flow expressions (9) and (16), respectively, to evaluate the lowest order radial plasma current (due to ion parallel viscosity). Dotting by $\boldsymbol{h}$ the sum of the electron and the ion momentum equations (with electron inertia and viscosity and ion cross-field viscosity neglected),

$$
M n(\boldsymbol{V} \cdot \nabla) \boldsymbol{V}=\frac{1}{c} \boldsymbol{J} \times \boldsymbol{B}-\nabla P-\nabla \cdot \overleftrightarrow{\pi}_{\|}
$$

and flux surface averaging gives in lowest order

$$
\frac{1}{c}\langle\boldsymbol{J} \cdot \nabla \chi\rangle=\left\langle\pi_{\|} \frac{\nabla_{\|}\left(u B^{2}\right)}{2 B}\right\rangle,
$$

where we have used

$$
\begin{array}{r}
\left\langle\boldsymbol{h} \cdot\left(\nabla \cdot \overleftrightarrow{\pi}_{\|}\right)\right\rangle=-\left\langle\overleftrightarrow{\pi}_{\|}: \nabla \boldsymbol{h}\right\rangle=-\left\langle\pi_{\|} \nabla_{\|} \boldsymbol{h} \cdot \hat{\boldsymbol{b}}\right\rangle= \\
\left\langle\pi_{\|}\left[\boldsymbol{\kappa} \cdot \boldsymbol{h}-\nabla_{\|}(u B)\right]\right\rangle=-\left\langle\pi_{\|} \frac{\nabla_{\|}\left(u B^{2}\right)}{2 B}\right\rangle
\end{array}
$$

Employing expression (20) for $\pi_{\|}$results in

$$
\frac{1}{c}\langle\boldsymbol{J} \cdot \nabla \chi\rangle=\frac{3}{4} c \eta G_{1}(\chi)\left(\frac{\partial \phi}{\partial \chi}+\frac{1}{e n} \frac{\partial p}{\partial \chi}\right)+c \eta\left[1.37 G_{1}(\chi)+0.085 G_{2}(\chi)\right] \frac{1}{e} \frac{\partial T}{\partial \chi},(26)
$$


where

$$
G_{1}(\chi) \equiv \frac{\left\langle\left(\nabla_{\|} \ln B\right) \nabla_{\|}\left(u B^{2}\right)\right\rangle^{2}}{\left\langle\left(\nabla_{\|} B\right)^{2}\right\rangle}-\left\langle\left[\frac{\nabla_{\|}\left(u B^{2}\right)}{B}\right]^{2}\right\rangle
$$

and

$$
G_{2}(\chi)=\left\langle u\left(\nabla_{\|} \ln B\right) \nabla_{\|}\left(u B^{2}\right)\right\rangle-\frac{\left\langle\left(\nabla_{\|} \ln B\right) \nabla_{\|}\left(u B^{2}\right)\right\rangle\left\langle u\left(\nabla_{\|} B\right)^{2}\right\rangle}{\left\langle\left(\nabla_{\|} B\right)^{2}\right\rangle} .
$$

It follows from the Schwartz inequality that $G_{1}(\chi)$ is always non-positive and vanishes if and only if

$$
B^{-1} \nabla_{\|}\left(u B^{2}\right)=F_{2}(\chi) \nabla_{\|} B
$$

with arbitrary $F_{2}(\chi)$. $u$ satisfying Eq. (29) also results in $G_{2}(\chi)=0$ and thereby $\langle\boldsymbol{J} \cdot \boldsymbol{\nabla} \chi\rangle=0$. It is clear from Eq. (15) that $\left.u\right|_{a s}$ satisfies constraint (29) and parallel ion viscosity does not contribute to the radial plasma current for an axisymmetric tokamak, as expected. Solving Eq. (29) for $u$ and plugging the result into Eq. (8) gives a coordinate-independent form of the necessary condition for intrinsically ambipolar particle transport in collisional plasmas confined by a three-dimensional toroidal magnetic field:

$$
\hat{\boldsymbol{b}} \times \boldsymbol{\kappa} \cdot \nabla \chi=F_{3}(\chi) \nabla_{\|} \ln B,
$$

with arbitrary $F_{3}(\chi)$. This agrees with arbitrary-collisionality findings of Ref. [16]. Moreover, Ref. [16] showed that condition (30) is equivalent to quasi-symmetry requirement for magnetic field in the Boozer coordinates $\left(\chi, \theta_{B}, \varphi_{B}\right)[15], B=$ $B\left(\chi, s \theta_{B}-t \varphi_{B}\right)$ with arbitrary integers $s$ and $t$.

\section{LIMIT OF A TOKAMAK WITH A SMALL TOROIDAL FIELD RIPPLE}

Next, we consider an almost axisymmetric collisional tokamak with a small toroidal field ripple and employ Eq. (26) to evaluate the contribution to the radial current due to the deviation from the axisymmetry. We use Hamada magnetic flux coordinates 
[40] $\left(v, \theta_{H}, \varphi_{H}\right)$, with $v$ the volume enclosed by a given magnetic flux surface and $\theta_{H}$ and $\varphi_{H}$ the poloidal and the toroidal angles, respectively, chosen such that $\boldsymbol{\nabla} v \times$ $\boldsymbol{\nabla} \theta_{H} \cdot \boldsymbol{\nabla} \varphi_{H}=1$. Then, the magnetic field can be conveniently written as

$$
\boldsymbol{B}=\boldsymbol{\nabla} \psi \times \nabla \theta_{H}+\nabla \varphi_{H} \times \nabla \chi=\psi^{\prime} \nabla v \times\left(\nabla \theta_{H}-\iota \nabla \varphi_{H}\right)=\psi^{\prime} \nabla v \times \nabla \alpha,
$$

with $\psi(v)$ and $\chi(v)$ the toroidal and the poloidal fluxes enclosed by the flux surface with volume $v, \psi^{\prime} \equiv \mathrm{d} \psi / \mathrm{d} v, \chi^{\prime} \equiv \mathrm{d} \chi / \mathrm{d} v, \iota \equiv \mathrm{d} \chi / \mathrm{d} \psi$, and $\alpha \equiv \theta_{H}-\iota \varphi_{H}$. It is often convenient to use $\left(v, \alpha, \varphi_{H}\right)$ coordinates instead of $\left(v, \theta_{H}, \varphi_{H}\right)$, and we will do so in this section. For example, in this case the parallel gradient has a particularly simple form: $\boldsymbol{B} \cdot \boldsymbol{\nabla}=\psi^{\prime}\left(\partial / \partial \varphi_{H}\right)$.

In the remaining portions of this work, we assume that the parallel length scale represents the characteristic distance along the magnetic field associated with the toroidal field ripple, $L_{\|} \sim 2 \pi R_{0} / N$, with $R_{0}$ the toroidal radius of the magnetic axis and the integer $N$ describing the ripple toroidal period. Therefore, the mean-free path expansion parameter $\Delta \sim N \lambda_{i} /\left(2 \pi R_{0}\right)$.

To proceed, we rewrite $G_{1}(\chi)$ and $G_{2}(\chi)$ in terms of $B$ and its $\alpha$ and $\varphi_{H}$ derivatives. First, we introduce a quantity $w \equiv \boldsymbol{B} \times \boldsymbol{\nabla} v \cdot \boldsymbol{\nabla} \varphi_{H}$ and notice that to order $\beta \Delta \delta \ll 1$ (i.e., neglecting effects of the ion parallel viscosity on the magnetic curvature)

$$
\frac{2}{B^{2} \iota} \hat{\boldsymbol{b}} \times \nabla \chi \cdot \boldsymbol{\kappa}=\frac{2}{B^{2} \iota} \hat{\boldsymbol{b}} \times \nabla \chi \cdot \nabla \ln B=-\nabla_{\|}\left(\frac{w}{B^{2}}\right)
$$

Then, Eq. (8) requires

$$
u=-\frac{w \iota}{B^{2}}+F_{4}(\chi)
$$

The arbitrary flux function $F_{4}$ cannot depend on $\alpha$ since it must be constant along field lines and, consequently, on entire rational and, by continuity, irrational flux surfaces. Moreover, since $u$ is defined up to an arbitrary flux function [see Eqs. (8) and (15)], we can choose $F_{4}=0$ without loss of generality. 
Employing relation $u=-w \iota / B^{2}$ in Eq. (8) we obtain

$$
\nabla_{\|}\left(u B^{2}\right)=\frac{\iota}{B} \frac{\partial B^{2}}{\partial \alpha}
$$

Noticing that

$$
\nabla_{\|} B=\frac{\psi^{\prime}}{B} \frac{\partial B}{\partial \varphi_{H}}
$$

and using the geometrical relations (34) and Eqs. (35) in (27) we find a convenient expression for $G_{1}$ :

$$
G_{1}(\chi)=\iota^{2}\left[\frac{\left\langle\left(\partial \ln B / \partial \varphi_{H}\right)\left(\partial \ln B^{2} / \partial \alpha\right)\right\rangle^{2}}{\left\langle\left(\partial \ln B / \partial \varphi_{H}\right)^{2}\right\rangle}-\left\langle\left(\frac{\partial \ln B^{2}}{\partial \alpha}\right)^{2}\right\rangle\right] .
$$

To evaluate $G_{2}(\chi)$ we first solve Eq. (34) for $u$ to obtain

$$
u B^{2}=\frac{\iota}{\psi^{\prime}} \int \mathrm{d} \varphi_{H} \frac{\partial B^{2}}{\partial \alpha}+F_{5}(\chi)
$$

As in Eq. (33), $F_{5}$ cannot depend on $\alpha$ and is therefore a flux function. Employing Eqs. (35) and (37) in Eq. (28) we arrive at the following expression for $G_{2}$ :

$$
\begin{array}{r}
G_{2}(\chi)=\iota^{2}\left[\left\langle\frac{1}{B^{2}}\left(\int \mathrm{d} \varphi_{H} \frac{\partial B^{2}}{\partial \alpha}+\frac{F_{5} \psi^{\prime}}{\iota}\right) \frac{\partial \ln B}{\partial \varphi_{H}} \frac{\partial \ln B^{2}}{\partial \alpha}\right\rangle\right. \\
\left.-\frac{\left\langle\left(\partial \ln B / \partial \varphi_{H}\right)\left(\partial \ln B^{2} / \partial \alpha\right)\right\rangle\left\langle B^{-2}\left[\int \mathrm{d} \varphi_{H}\left(\partial B^{2} / \partial \alpha\right)+F_{5} \psi^{\prime} / \iota\right]\left(\partial \ln B / \partial \varphi_{H}\right)^{2}\right\rangle}{\left\langle\left(\partial \ln B / \partial \varphi_{H}\right)^{2}\right\rangle}\right]
\end{array}
$$

Concentrating on the limit of large-aspect-ratio tokamak with circular cross-section and small toroidal ripple, we next assume [41]

$$
B\left(r, \alpha, \varphi_{H}\right)=B_{0}(r)\left[1-\epsilon(r) \cos \left(\alpha+\iota \varphi_{H}\right)-\rho(r) \cos \left(N \varphi_{H}\right)+O\left(\epsilon^{2}, \rho^{2}, \epsilon \rho\right)\right]
$$

where $r$ is the poloidal radius of a flux surface, $\epsilon \equiv r / R_{0} \ll 1$ is the inverse aspect ratio of the flux surface, and $\rho \ll 1$ is a measure of the magnitude of the ripple. Substituting expression (39) into Eqs. (36) and (38) for $G_{1}$ and $G_{2}$ and noticing that $\langle\cdots\rangle=(2 \pi)^{-2} \oint \mathrm{d} \alpha \mathrm{d} \varphi_{H}(\cdots)$ we obtain to lowest order in $\epsilon$ and $\rho$

$$
G_{1}(\chi)=-\frac{2(N \rho)^{2}(\epsilon \iota)^{2}}{(N \rho)^{2}+(\epsilon \iota)^{2}}
$$


and, taking into account that $N^{2} \gg 1 \gtrsim \iota^{2}$,

$$
G_{2}(\chi)=\frac{(N \rho)^{2}(\epsilon \iota)^{2}}{(N \rho)^{2}+(\epsilon \iota)^{2}}\left[\left(\frac{7}{4} \epsilon^{2}-\rho^{2}\right)-\frac{7}{4} \frac{F_{5} \psi^{\prime}}{B_{0}^{2}}\left(\epsilon^{2}-\rho^{2}\right)\right]
$$

Employing expansion (39) in Eq. (37) and comparing with Eq. (15) gives that $F_{5} \psi^{\prime} / B_{0}^{2}$ is at most of order unity in the $\epsilon$ and $\rho$ expansion, so that $\left|G_{2}\right| \ll\left|G_{1}\right|$. Since $\partial / \partial \chi=\left(B_{0} \iota r\right)^{-1} \partial / \partial r$, the lowest order radial current from Eq. (26) is

$$
\langle\boldsymbol{J} \cdot \nabla \chi\rangle_{\text {ripple }}=-\frac{c^{2} p}{\nu \iota B_{0}} \frac{(N \rho)^{2}(\epsilon \iota)^{2}}{(N \rho)^{2}+(\epsilon \iota)^{2}} \frac{1}{r}\left[1.44\left(\frac{\partial \phi}{\partial r}+\frac{1}{e n} \frac{\partial p}{\partial r}\right)+2.63 \frac{1}{e} \frac{\partial T}{\partial r}\right] .
$$

In reality, the ripple magnitude is usually a strong function of the poloidal angle, reaching maximum about the tokamak outer midplane, so that $\rho(r)$ must be replaced by $\rho\left(r, \theta_{H}\right)$ in Eq. (39). This leads to replacements $\rho^{2} \rightarrow\left\langle\rho^{2}\right\rangle$ and $\epsilon^{2} \rightarrow \epsilon^{2}+\left\langle\left(\partial \rho / \partial \theta_{H}\right)^{2}\right\rangle$ in Eq. (42), with the latter replacement seemingly unimportant.

\section{RADIAL ELECTRIC FIELD}

Having evaluated the contribution to the radial current caused by the small toroidal field ripple, we can now estimate how big should the ripple be to influence the radial electric field and plasma rotation in a rippled tokamak. This is done by setting the total radial current to zero and comparing "the ripple" and "the axisymmetric" contributions to the radial electric field.

The toroidal field ripple contribution to the radial current, as given by Eq. (42), is proportional to the ripple magnitude squared and vanishes in the axisymmetric limit. Then, radial current is dominated by cross-field ion viscosity contributions [3]. Such contributions have been evaluated in Ref. [6-9]. In the limit of $B_{P} / B_{T} \ll 1$, where $B_{P}$ and $B_{T}$ are the magnitudes of the poloidal and the toroidal components of $\boldsymbol{B}$, respectively, we can obtain for axisymmetric tokamaks with up-down symmetric but 
otherwise arbitrary poloidal cross-sections [7]:

$$
\begin{array}{r}
\langle\boldsymbol{J} \cdot \nabla \chi\rangle_{\text {axisym }}=\frac{1.2}{v^{\prime}} \frac{\partial}{\partial \chi}\left[v^{\prime} \frac{c^{2} p}{\nu}\left(\frac{\nu B}{\Omega}\right)^{2}\left\langle\frac{R^{4} B_{P}^{2}}{B^{2}}\right\rangle \frac{\partial}{\partial \chi}\left(\frac{\partial \phi}{\partial \chi}+\frac{1}{e n} \frac{\partial p}{\partial \chi}\right)\right] \\
-\frac{0.23}{v^{\prime}} \frac{\partial}{\partial \chi}\left[v^{\prime} \frac{c^{2} p}{\nu} \frac{I^{2} \nu^{2}}{e\left\langle\Omega^{2}\right\rangle} \frac{T T_{e}}{T+T_{e}}\left(\left\langle R^{2}\right\rangle-\frac{\left\langle R^{2} B^{2}\right\rangle}{\left\langle B^{2}\right\rangle}\right)\left(\frac{\partial \ln T}{\partial \chi}\right)^{2}\right] \\
+\frac{0.64}{v^{\prime}} \frac{\partial}{\partial \chi}\left\{v^{\prime} \frac{\partial}{\partial \chi}\left[\frac{c^{2} p}{\nu} \frac{I^{2} \nu^{2} B^{2}}{e \Omega^{2}}\left(2\left\langle R^{2}\right\rangle\left\langle\frac{1}{B^{2}}\right\rangle-\frac{\left\langle R^{2}\right\rangle}{\left\langle B^{2}\right\rangle}-\left\langle\frac{R^{2}}{B^{2}}\right\rangle\right) \frac{\partial T}{\partial \chi}\right]\right\},
\end{array}
$$

where $v^{\prime} \equiv \mathrm{d} v / \mathrm{d} r=\oint(\mathrm{d} \theta / \boldsymbol{B} \cdot \boldsymbol{\nabla} \theta)$ with $\theta$ the poloidal angle. Expression (43) can be further simplified by considering the large aspect-ratio circular poloidal crosssection limit (see e.g. Ref. [42]): $R=R_{0}(1+\epsilon \cos \theta), B_{T}=B_{0} /(1+\epsilon \cos \theta)$, and $B_{P}=\epsilon B_{0} \iota /(1+\Lambda \epsilon \cos \theta)$, where $\Lambda \equiv 1-\epsilon^{-1} \partial \Delta_{s} / \partial r$ with $\Delta_{s}$ the Shafranov shift. Then, to lowest order in $\epsilon$,

$$
\begin{array}{r}
\langle\boldsymbol{J} \cdot \nabla \chi\rangle_{\text {axisym }}=\frac{1}{r} \frac{\partial}{\partial r}\left\{\frac { c ^ { 2 } p } { \nu \iota B _ { 0 } } ( \frac { \nu } { \Omega _ { 0 } } ) ^ { 2 } R _ { 0 } ^ { 4 } \epsilon ^ { 2 } \left[1.2 \frac{\iota}{r} \frac{\partial}{\partial r}\left(\frac{1}{\iota r} \frac{\partial \phi}{\partial r}+\frac{1}{e n \iota r} \frac{\partial p}{\partial r}\right)\right.\right. \\
\left.\left.-0.45\left(\frac{T_{e}}{T+T_{e}}\right) \frac{1}{\iota^{2} r^{2}} \frac{\partial \ln T}{\partial r}\left(\frac{1}{e} \frac{\partial T}{\partial r}\right)\right]\right\},
\end{array}
$$

where $\Omega_{0} \equiv e B_{0} /(M c)$.

When effects of the ripple are negligible, radial electric field, $E_{r} \equiv-\partial \phi / \partial r$, is determined by requiring $\langle\boldsymbol{J} \cdot \boldsymbol{\nabla} \chi\rangle_{\text {axisym }}=0[6,7]$ :

$$
\frac{\partial}{\partial r}\left[\frac{1}{\iota r}\left(\frac{\partial \phi}{\partial r}+\frac{1}{e n} \frac{\partial p}{\partial r}\right)\right]=0.38\left(\frac{T_{e}}{T+T_{e}}\right) \frac{1}{\iota^{3} r} \frac{\partial \ln T}{\partial r}\left(\frac{1}{e} \frac{\partial T}{\partial r}\right) .
$$

When effects of the ripple dominate, $E_{r}$ is determined by requiring $\langle\boldsymbol{J} \cdot \nabla \chi\rangle_{\text {ripple }}=0$ and Eq. (42) gives

$$
\frac{\partial \phi}{\partial r}+\frac{1}{e n} \frac{\partial p}{\partial r}=-1.83 \frac{1}{e} \frac{\partial T}{\partial r} .
$$

Using this to evaluate the toroidal component of ion flow from Eq. (16) gives $n \boldsymbol{V}$. $R \boldsymbol{\nabla} \zeta=0$, i.e. the toroidal flow is damped, in agreement with Ref. [30].

In general, for ripple small enough that the radial current contribution (44) due to cross-field ion viscosity, derived for axisymmetric magnetic field, is approximately 
valid, $E_{r}$ must be determined from Eqs. (42) and (44) by requiring $\langle\boldsymbol{J} \cdot \nabla \chi\rangle_{\text {ripple }}+$ $\langle\boldsymbol{J} \cdot \boldsymbol{\nabla} \chi\rangle_{\text {axisym }}=0$. Comparing coefficients in front of $E_{r}$ in Eqs. (42) (with $\rho^{2} \rightarrow\left\langle\rho^{2}\right\rangle$ ) and (44) we see that ripple effects are important when

$$
\frac{N}{2 \pi} \frac{\delta}{\Delta}<\sqrt{\frac{N^{2}\left\langle\rho^{2}\right\rangle(\epsilon \iota)^{2}}{N^{2}\left\langle\rho^{2}\right\rangle+(\epsilon \iota)^{2}}} \sim \min \left[\epsilon \iota, N\left\langle\rho^{2}\right\rangle^{1 / 2}\right]
$$

and that these effects are negligible when the opposite inequality is satisfied, where $\delta=\rho_{i} / L_{\perp}$ and $\Delta=N \lambda_{i} /\left(2 \pi R_{0}\right)$.

Assuming that the characteristic perpendicular length scale is determined by the pedestal width of order the poloidal ion gyroradius, $L_{\perp} \sim \rho_{p i} \equiv v_{T i} M c /\left(e B_{P}\right)$, as it may be in the H-mode regime, we obtain $\delta \sim B_{P} / B_{T} \sim \epsilon \iota$. Inequality (47) becomes then

$$
\frac{2 \pi \Delta}{N}>\sqrt{1+\frac{\epsilon^{2} \iota^{2}}{N^{2}\left\langle\rho^{2}\right\rangle}}
$$

Since $\Delta \ll 1$ and $2 \pi / N \lesssim 1$ (e.g. $N=20$ for Alcator C-Mod [43]), inequality (48) is never satisfied and ripple effects on plasma rotation in this case are expected to be negligible. However, it is conceivable that such effects may be important in the L-mode regime, where $L_{\perp} \sim r \gg \rho_{p i}$.

\section{CONCLUSIONS}

We have considered the neoclassical equilibrium of a short mean-free path plasma confined by an arbitrary three-dimensional toroidal magnetic field, and calculated the rotation velocity and the non-ambipolar radial current. The former is given by Eqs. (16), (18) and (21), and the latter by Eqs. (26) - (28). This has allowed us to obtain the necessary condition for intrinsically ambipolar particle transport in such a field, given by Eq. (30), which agrees with the arbitrary-collisionality result of Ref. [16]. 
We have also employed the general expression for this non-ambipolar radial current to obtain an estimate for a tokamak with a small toroidal ripple, Eq. (42), and to estimate the ripple size that can affect the axisymmetric plasma rotation result of Refs. [6-9]. We have found that the ripple effects dominate when inequality (47) is satisfied and are unimportant otherwise. If the characteristic perpendicular length scale is given by the pedestal width of order the ion poloidal gyroradius (as might be the case in the H-mode regime), inequality (47) simplifies and is replaced by Eq. (48). However, this inequality is never satisfied for short mean-free path plasmas, meaning that toroidal field ripple effects on plasma rotation are expected to be unimportant in this case. On the other hand, the ripple effects may become important for more shallow plasma gradients.

An interesting and potentially important effect that was not accounted for in this paper is the non-linear back-reaction of the radial electric field on the radial transport. In our ordering scheme, this effect is formally small. In tokamaks, it was shown by Hinton and Wong $[12,44]$ that this effect becomes important when the Mach number squared, $\left(V / v_{T i}\right)^{2}$, is comparable to our parameter $\Delta$, defined by Eq. (1), but no rigorous calculation has to our knowledge been performed for the case of stellarator geometry. In addition, we did not consider the dynamical process of approaching the ambipolar state $[14,32]$. We also neglected effects of neutral particles on momentum transport [32], which can become important for neutral fractions of order $0.1 \%$ or smaller [45].

\section{ACKNOWLEDGEMENT}

The authors thank M. Greenwald and S. M. Wolfe for information on the Alcator C-Mod toroidal field ripple and P. J. Catto for helpful comments. This work was 
supported in part by the U.S.A. Department of Energy grant DE-AC52-06NA-25396 at Los Alamos National Laboratory.

\section{APPENDIX A: WHY REF. [29] DOES NOT RECOVER THE CORRECT AXISYMMETRIC TOKAMAK AND QUASI-SYMMETRIC STELLARATOR LIMITS}

This appendix discusses why Ref. [29] failed to reproduce the necessary condition (30) for intrinsically ambipolar particle transport in collisional plasmas confined by a three-dimensional toroidal magnetic field.

It is convenient to start from the well-known drift-kinetic expression for a radial particle flux (the equation right after Eq. (A26) of Ref. [29]),

$$
\left\langle n_{j} \boldsymbol{V}_{j} \cdot \nabla \chi\right\rangle=\left\langle\int \mathrm{d}^{3} v\left(\boldsymbol{v}_{d j} \cdot \nabla \chi\right) \bar{f}_{j}\right\rangle,
$$

where $j=e, i$ denotes particle species, $\bar{f}_{j}$ is a gyrophase-averaged distribution function accurate to first order in the gyroradius expansion, and

$$
\boldsymbol{v}_{d j} \equiv-v_{\|} \hat{\boldsymbol{b}} \times \nabla_{U, \mu}\left(\frac{v_{\|}}{\Omega_{j}}\right)
$$

is the lowest order perpendicular drift velocity with $\Omega_{j} \equiv e_{j} B /\left(M_{j} c\right)$ the species' gyrofrequency and $\boldsymbol{\nabla}_{U, \mu}$ evaluated holding the total energy $U=v_{\|}^{2} / 2+\mu B+e_{j} \phi / M_{j}$ and magnetic moment $\mu=v_{\perp}^{2} /(2 B)$ fixed. Substituting $\boldsymbol{v}_{d j}$ gives

$$
\left\langle n_{j} \boldsymbol{V}_{j} \cdot \boldsymbol{\nabla} \chi\right\rangle=\frac{c}{e_{j}}\left\langle\left(p_{\| j}+p_{\perp j}\right) \frac{\hat{\boldsymbol{b}} \times \boldsymbol{\kappa} \cdot \boldsymbol{\nabla} \chi}{B}\right\rangle+c\left\langle n_{j} \frac{\hat{\boldsymbol{b}} \times \boldsymbol{\nabla} \phi \cdot \boldsymbol{\nabla} \chi}{B}\right\rangle
$$

with $p_{\| j}=M_{j} \int \mathrm{d}^{3} v v_{\|}^{2} \bar{f}_{j}$ and $p_{\perp j}=M_{j} \int \mathrm{d}^{3} v \mu B \bar{f}_{j}$, so that accounting for quasineutrality $\sum_{j} e_{j} n_{j}=0$ produces the following convenient expression for the radial current,

$$
\frac{1}{c}\langle\boldsymbol{J} \cdot \nabla \chi\rangle=\left\langle\sum_{j}\left(p_{\| j}+p_{\perp j}\right) \frac{\hat{\boldsymbol{b}} \times \boldsymbol{\kappa} \cdot \boldsymbol{\nabla} \chi}{B}\right\rangle,
$$


in agreement with Ref. [29].

Expanding $\bar{f}_{j}$ into a series of Legendre polynomials $P_{k}\left(v_{\|} / v\right)$ and noticing that to the order required $k=0,1,2$ we obtain

$$
\bar{f}_{j}=\sum_{k=0}^{2} \bar{f}_{j}^{(k)}(v) P_{k}\left(v_{\|} / v\right)
$$

Introducing $p_{\| j}^{(k)} \equiv M_{j} \int \mathrm{d}^{3} v v_{\|}^{2} \bar{f}_{j}^{(k)} P_{k}\left(v_{\|} / v\right)$ and $p_{\perp j}^{(k)} \equiv M_{j} \int \mathrm{d}^{3} v \mu B \bar{f}_{j}^{(k)} P_{k}\left(v_{\|} / v\right)$, noticing that $p_{\| j}^{(0)}=p_{\perp j}^{(0)}$ and $p_{\| j}^{(1)}=p_{\perp j}^{(1)}=0$, and employing Eq. (8) gives

$$
\frac{1}{c}\langle\boldsymbol{J} \cdot \nabla \chi\rangle=-\left\langle\sum_{j} p_{\| j}^{(0)} \boldsymbol{B} \cdot \nabla u+\sum_{j}\left(p_{\| j}^{(2)}+p_{\perp j}^{(2)}\right) \frac{\boldsymbol{B} \cdot \boldsymbol{\nabla} u}{2}\right\rangle .
$$

Finally, employing the parallel velocity moment of a first-order accurate drift-kinetic equation (see for example Eq. (38) of Ref. [36]),

$$
\boldsymbol{B} \cdot \boldsymbol{\nabla}\left(p_{\| j}^{(0)}+p_{\| j}^{(2)}\right)=\left(p_{\| j}^{(2)}-p_{\perp j}^{(2)}\right) \nabla_{\|} B
$$

in Eq. (A6) gives

$$
\frac{1}{c}\langle\boldsymbol{J} \cdot \nabla \chi\rangle=\left\langle\sum_{j}\left(p_{\| j}^{(2)}-p_{\perp j}^{(2)}\right) \frac{\nabla_{\|}\left(u B^{2}\right)}{2 B}\right\rangle=\left\langle\sum_{j}\left(p_{\| j}-p_{\perp j}\right) \frac{\nabla_{\|}\left(u B^{2}\right)}{2 B}\right\rangle .
$$

Since electron pressure anisotropy is small and can be neglected compared with that of ions,

$$
\frac{1}{c}\langle\boldsymbol{J} \cdot \nabla \chi\rangle \approx\left\langle\left(p_{\| i}-p_{\perp i}\right) \frac{\nabla_{\|}\left(u B^{2}\right)}{2 B}\right\rangle .
$$

Expression (A9) for the radial current coincides with Eq. (24) obtained from the fluid approach. At the same time, it disagrees with the expression used in Ref. [29], Eq. (A27), which unjustifiably approximates $\nabla_{\|}\left(u B^{2}\right) / 2 B=(\boldsymbol{B} \cdot \boldsymbol{\nabla} u) / 2+u \nabla_{\|} B$ with $(\boldsymbol{B} \cdot \boldsymbol{\nabla} u) / 2$. As a result of this approximation, Ref. [29] obtains an incorrect ambipolarity condition [compare with Eq. (30)]

$$
\hat{\boldsymbol{b}} \times \boldsymbol{\kappa} \cdot \nabla \chi=F_{6}(\chi) \nabla_{\|} B^{2}
$$


with $F_{6}(\chi)$ an arbitrary flux function. Moreover, contrary to the claim made in Ref. [29], their radial current does not vanish in the axisymmetric limit, where $\hat{\boldsymbol{b}} \times$ $\boldsymbol{\kappa} \cdot \nabla \chi=-I(\chi) \nabla_{\|} \ln B$

[1] K. H. Burrell, T. N. Carlstrom, E. J. Doyle, D. Finkenthal, P. Gohil, R. J. Groebner, D. L. Hillis, J. Kim, H. Matsumoto, R. A. Moyer, T. H. Osborne, C. L. Rettig, W. A. Peebles, T. L. Rhodes, H. StJohn, R. D. Stambaugh, M. R. Wade, and J. G. Watkins, Plasma Phys. Control. Fusion 34, 1859 (1992) and references therein.

[2] P. H. Rutherford, Phys. Fluids 13, 482 (1970).

[3] F. L. Hinton and R. D. Hazeltine, Rev. Mod. Phys. 48, 239 (1976).

[4] A. N. Simakov and P. J. Catto, Plasma Phys. Control. Fusion 49, 729 (2007).

[5] R. D. Hazeltine and J. D. Meiss, Plasma Confinement (Addison-Wesley, Redwood City CA, 1991), p. 178.

[6] H. A. Claassen, H. Gerhauser, A. Rogister, and C. Yarim, Phys. Plasmas 7, 3699 (2000).

[7] P. J. Catto and A. N. Simakov, Phys. Plasmas 12, 012501 (2005).

[8] S. K. Wong and V. S. Chan, Phys. Plasmas 14, 112505 (2007).

[9] S. K. Wong and V. S. Chan, Phys. Plasmas 14, 122501 (2007).

[10] M. N. Rosenbluth, P. H. Rutherford, J. P. Taylor, E. A. Frieman, and L. M. Kovrizhnykh, Plasma Phys. Controlled Nucl. Fusion Res. 1, 495 (1971).

[11] S. K. Wong and V. S. Chan, Phys. Plasmas 12, 092513 (2005).

[12] F. L. Hinton and S. K. Wong, Phys. Fluids 28, 3082 (1985).

[13] P. J. Catto, I. B. Bernstein, and M. Tessarotto, Phys. Fluids 30, 2784 (1987).

[14] K. C. Shaing, Phys. Fluids 29, 2231 (1986). 
[15] A. H. Boozer, Phys. Fluids 26, 496 (1983).

[16] P. Helander and A. N. Simakov, Phys. Rev. Lett. 101, 145003 (2008).

[17] J. Nührenberg, W. Lotz, and S. Gori, Theory of Fusion Plasmas (Editrice Compositori, Bologna, 1994), p. 3.

[18] J. Nührenberg and R. Zille, Phys. Lett. A 129, 113 (1988).

[19] K. C. Shaing, S. P. Hirshman, and J. D. Callen, Phys. Fluids 29, 521 (1986).

[20] D. D.-M. Ho and R. M. Kulsrud, Phys. Fluids 30, 442 (1987).

[21] A. B. Mikhailovskii, Plasma Phys. Rep. 21, 529 (1995).

[22] B. N. Kuvshinov, A. B. Mikhailovskii, and S. E. Sharapov, Plasma Phys. Rep. 21, 713 (1995).

[23] K. C. Shaing, Phys. Rev. Lett. 76, 4364 (1996).

[24] L. M. Kovrizhnykh, Comments Plasma Phys. Control. Fusion 18, 309 (1998).

[25] L. M. Kovrizhnykh, Plasma Phys. Rep. 25, 760 (1999).

[26] K. C. Shaing, Phys. Plasmas 13, 052505 (2006).

[27] J. D. Callen, A. J. Cole, and C. C. Hegna, 22nd IAEA Fusion Energy Conference, Geneva, Switzerland (International Atomic Energy Agency, Vienna, 2009), paper IAEA-CN-165/TH/P8-36.

[28] L. M. Kovrizhnykh, Nucl. Fusion 24, 851 (1984).

[29] K. T. Tsang and E. A. Frieman, Phys. Fluids 19, 747 (1976).

[30] A. B. Mikhailovskii and V. S. Tsypin, Sov. J. Plasma Phys. 10, 142 (1984).

[31] K. C. Shaing and J. D. Callen, Phys. Fluids 26, 3315 (1983).

[32] M. Coronado and J. N. Talmadge, Phys. Fluids B 5, 1200 (1993).

[33] H. Wobig and J. Kisslinger, Plasma Phys. Control. Fusion 37, 893 (1995).

[34] V. S. Tsypin, A. B. Mikhailovskii, R. M. O. Galvaõ, I. C. Nascimento, M. Tendler, C. A. de Azevedo, and A. S. de Assis, Phys. Plasmas 5, 3358 (1998). 
[35] S. P. Hirshman and D. J. Sigmar, Nucl. Fusion 21, 1079 (1981).

[36] R. D. Hazeltine, Phys. Fluids 17, 961 (1974) with the following corrections: (i) 0.27 replaced with 0.05 in Eqs. (60) and (61); (ii) 3.1 replaced with 2.9 in Eq. (67). (iii) -2.1 replaced with -1.9 in the $\Delta \ll 1$ form of Eq. (69); and (iv) the slash removed in $S_{1}^{0}$ between Eqs. (51) and (52) so it reads as $S_{1}^{0}=x^{3}+2 x^{5}$.

[37] S. I. Braginskii, in Reviewes of Plasma Physics, edited by M. A. Leontovich (Consultants Bureau, New York, 1965), Vol. 1, p. 205.

[38] P. J. Catto and A. N. Simakov, Phys. Plasmas 11, 90 (2004); ibid. 12, 114503 (2005).

[39] A. N. Simakov and P. J. Catto, Phys. Plasmas 10, 4744 (2003).

[40] S. Hamada, Nucl. Fusion 2, 23 (1962).

[41] J. W. Connor and R. J. Hastie, Nucl. Fusion 13, 221 (1973).

[42] P. Helander and D. J. Sigmar, Collisional Transport in Magnetized Plasmas (Cambridge University Press, Cambridge, 2002), p. 127.

[43] I. H. Hutchinson, R. Boivin, F. Bombarda, P. Bonoli, S. Fairfax, C. Fiore, J. Goetz, S. Golovato, R. Granetz, M. Greenwald, S. Horne, A. Hubbard, J. Irby, B. LaBombard, B. Lipschultz, E. Marmar, G. McCracken, M. Porkolab, J. Rice, J. Snipes, Y. Takase, J. Terry, S. Wolfe, C. Christensen, D. Garnier, M. Graf, T. Hsu, T. Luke, M. May, A. Niemczewski, G. Tinios, J. Schachter, and J. Urbahn, Phys. Plasmas 1, 1511 (1994).

[44] S. K. Wong and F. L. Hinton, Phys. Rev. Lett. 52, 827 (1984).

[45] P. J. Catto, P. Helander, J. W. Connor, and R. D. Hazeltine, Phys. Plasmas 5, 3961 (1998). 